\title{
Análise da Revisão Cochrane: Suplementação Alimentar com Ácidos Gordos Ómega-3 no Tratamento da Demência. Cochrane Database Syst Rev. 2016;4:CD009002.
}

\author{
Analysis of the Cochrane Review: Omega-3 Fatty Acids for \\ the Treatment of Dementia. Cochrane Database Syst Rev. \\ 2016;4:CD009002.
}

David Cordeiro SOUSA* $\bigotimes^{1,2}$, Guilherme Ferreira Dos SANTOS*3,4, João COSTA ${ }^{4,6}$, António VAZ-CARNEIRO ${ }^{4,5,6}$

Acta Med Port 2017 Oct;30(10):671-674 - https://doi.org/10.20344/amp.9743

\begin{abstract}
RESUMO
A suplementação alimentar com ácidos gordos poli-insaturados ómega-3, de origem animal e vegetal, é comummente prescrita como alternativa não farmacológica no tratamento da demência, com o objetivo de atrasar a sua progressão e melhorar a função cognitiva dos doentes. Esta utilização deriva em grande medida de estudos pré-clínicos, que estabeleceram o papel dos ácidos gordos poliinsaturados ómega-3 no desenvolvimento e manutenção da integridade do sistema nervoso central, e de estudos epidemiológicos, que demonstraram a ocorrência de estados de malnutrição em doentes com demência. Nesta revisão sistemática Cochrane, os autores incluíram três ensaios clínicos aleatorizados controlados por placebo, com baixo risco de vieses, nos quais doentes com doença de Alzheimer ligeira a moderada foram tratados com ácidos gordos poli-insaturados ómega-3, através de suplementos alimentares. Dos principais resultados desta RS destacam-se a ausência de evidência de um efeito benéfico do tratamento com suplementação com ácidos gordos poli-insaturados ómega-3 sobre a função cognitiva de doentes com doença de Alzheimer e a baixa taxa de efeitos adversos reportados, esta última comparável à dos grupos tratados com placebo. Não existem dados sobre outros tipos de demência. Neste artigo sumarizam-se e discutem-se os principais resultados e conclusões desta revisão sistemática, bem como as suas implicações para a prática clínica diária.
\end{abstract}

Palavras-chave: Ácidos Gordos Ómega-3; Demência; Ensaios Clínicos Aleatorizados; Revisão Sistemática

\section{ABSTRACT}

Dietary supplementation with omega-3 polyunsaturated fatty acids from fish and plant sources is commonly prescribed as a nonfarmacological alternative to improve brain functions and slow down the progression of dementia. This use is mostly based on findings of preclinical studies which established the role of omega-3 polyunsaturated fatty acids in the development and integrity of the brain, as well as epidemiological research that found evidence of malnutrition in patients with dementia. This Cochrane systematic review included three randomized, placebo-controlled trials at low risk of bias, in which omega-3 polyunsaturated fatty acids were administered to people with mild to moderate Alzheimer's disease in the form of supplements. Of the main results of this systematic review we highlight the lack of convincing evidence for the efficacy of omega-3 polyunsaturated fatty acids supplementation in the treatment of patients with Alzheimer's disease, as well as the low frequency of reported adverse events, with a comparable overall frequency between omega-3 polyunsaturated fatty acids and the placebo groups. The effects on other populations with dementia remain unclear. This paper aims to summarize and discuss the main results and conclusions of this systematic review, as well as its implications for the daily clinical practice.

Keywords: Omega-3 Fatty Acids; Dementia; Randomized Controlled Trials; Systematic Review

\section{QUESTÃO CLÍNICA}

Existe evidência científica disponível para suportar a suplementação com ácidos gordos poli-insaturados (AG) ómega-3 no tratamento da demência?

\section{OBJECTIVOS}

Avaliar e analisar a evidência científica atualmente disponível sobre a eficácia e segurança da suplementação com AG ómega-3 no tratamento da demência.

\section{METODOLOGIA}

A pesquisa foi efectuada no Specialized Register of the
Cochrane Dementia and Cognitive Improvement Group (ALOIS), MEDLINE, EMBASE, PsycINFO, CINAHL, ClinicalTrials.gov e no portal da Organização Mundial de Saúde [World Health Organization (WHO)], até 10 de dezembro de 2015. Foram ainda contactadas companhias farmacêuticas e organizações nacionais e internacionais, responsáveis pela produção, comercialização, controlo e certificação de suplementos alimentares de AG ómega-3, de modo a identificar ensaios clínicos a decorrer à data, ou ainda não publicados.

Nesta revisão sistemática (RS), os autores incluíram ensaios clínicos aleatorizados (ECAs) controlados por

\footnotetext{
* Co-primeiros autores.

1. Serviço de Oftalmologia. Hospital de Santa Maria. Centro Hospitalar de Lisboa Norte. Lisboa. Portugal.

2. Centro de Estudos das Ciências da Visão. Faculdade de Medicina. Universidade de Lisboa. Lisboa. Portugal.

3. Departamento de Medicina Física e Reabilitação. Hospital de Curry Cabral. Centro Hospitalar de Lisboa Central. Lisboa. Portugal.

4. Centro de Estudos de Medicina Baseada na Evidência. Faculdade de Medicina. Universidade de Lisboa. Lisboa. Portugal.

5. Instituto de Medicina Preventiva e Saúde Pública. Faculdade de Medicina. Universidade de Lisboa. Lisboa. Portugal.

6. Centro Colaborador Português da Rede Cochrane Iberoamericana. Faculdade de Medicina. Universidade de Lisboa. Lisboa. Portugal.

$\triangle$ Autor correspondente: David Cordeiro Sousa. davidscsousa@gmail.com.

Recebido: 28 de setembro de 2017 - Aceite: 12 de outubro de 2017 | Copyright @ Ordem dos Médicos 2017
} 
placebo, nos quais doentes com doença de Alzheimer (DA), demência vascular (DV), demência com corpos de Lewy (DCL), doença de Parkinson (DP) e demência fronto-temporal (DFT) foram tratados com suplementos alimentares (em que o principal ingrediente ativo era um AG ómega-3) ou através de uma dieta enriquecida em AG ómega-3. Foram considerados todos os ECAs em que a dosagem de AG ómega-3 administrada foi regular (pelo menos uma vez por semana) e a duração total de tratamento igual ou superior a 26 semanas.

As principais medidas de eficácia estudadas foram alterações globais e específicas da função cognitiva, medidas de acordo com escalas internacionais validadas, e a ocorrência de eventos adversos (ver Tabela 1).

As medidas secundárias de eficácia estudadas foram o efeito do tratamento com AG ómega-3 sobre a qualidade de vida; a adesão ao tratamento; a variação dos sintomas associados à demência (alterações do humor, alterações no ritmo circadiano); o número de internamentos hospitalares ocorridos e a taxa de mortalidade.

Foi realizada uma meta-análise de efeitos fixos para estimar o resultado médio aos seis meses da suplementação com AG ómega-3 nas várias medidas de eficácia em doentes com demência.

\section{RESULTADOS}

Esta RS incluiu três ECAs controlados por placebo, que investigaram o efeito da suplementação de AG ómega-3 num total de 632 participantes com DA ligeira a moderada, com avaliação aos seis, doze e dezoito meses. Os autores não encontraram ECAs que investigassem o efeito da suplementação com AG ómega-3 sobre outros tipos de demência. Todos os ECAs incluídos nesta RS apresentaram elevada qualidade metodológica. De forma global, a qualidade da evidência científica disponível para a maioria das medidas de eficácia estudadas foi alta.

Não foi detectado nenhum benefício da suplementação com AG ómega-3 sobre a função cognitiva, quando avaliada aos seis meses através da Alzheimer's Disease Assessment Scale - Cognitive subscale (ADAS-Cog) [diferença média padronizada (DMP) de $-0,02$, intervalo de confiança (IC) de $95 \%$ de $-0,19$ a 0,15; 566 participantes; três estudos; evidência científica de alta qualidade] ou do Mini-Mental State Examination (MMSE) [diferença média (DM) de 0,18, IC 95\% de $-1,05$ a 1,41; 202 participantes; 2 estudos; evidência científica de alta qualidade], ou nas atividades da vida diária [DM de $-0,02$; IC $95 \%$ de $-0,19$ a 0,$16 ; 544$ participantes; dois estudos; evidência científica de alta qualidade]. Da mesma forma, não se detectou um efeito

Tabela 1 - Ácidos gordos poli-insaturados ómega-3 em comparação com placebo na DA ligeira a moderada

População: Doentes com DA ligeira a moderada

Intervenção: Suplementos de ácidos gordos poli-insaturados ómega-3

Comparador: Placebo

\begin{tabular}{|c|c|c|c|c|c|}
\hline Medidas avaliadas & \multicolumn{2}{|c|}{$\begin{array}{l}\text { Efeito absoluto antecipado* (95\% IC) } \\
\text { Risco de doença de Alzheimer ligeira a moderada }\end{array}$} & $\begin{array}{l}\text { Efeito } \\
\text { relativo } \\
(95 \% \text { IC) }\end{array}$ & $\begin{array}{l}\text { Número de } \\
\text { participantes } \\
\text { (estudos) }\end{array}$ & $\begin{array}{l}\text { Qualidade } \\
\text { da evidência } \\
(\text { GRADE)** }\end{array}$ \\
\hline $\begin{array}{l}\text { Qualquer evento adverso } \\
\text { (combinado: diarreia, infeção } \\
\text { do trato urinário, quedas, } \\
\text { agitação) } \\
\text { Follow-up: } 18 \text { meses }\end{array}$ & 878 por 1000 & $\begin{array}{l}896 \text { por } 1000 \\
(834 \text { a } 966)\end{array}$ & $\begin{array}{l}\text { RR } 1,02 \\
(0,95 \text { a } 1,10)\end{array}$ & $402(1)$ & Moderada $^{1}$ \\
\hline $\begin{array}{l}\text { Eventos adversos graves } \\
\text { "definidos como eventos } \\
\text { que resultam em } \\
\text { morte, hospitalização } \\
\text { ou prolongamento do } \\
\text { internamento" } \\
\text { Follow-up: } 18 \text { meses }\end{array}$ & 305 por 1000 & $\begin{array}{l}320 \text { por } 1000 \\
(238 \text { a } 430)\end{array}$ & $\begin{array}{l}\text { RR 1,05 } \\
(0,78 \text { a } 1,41)\end{array}$ & $402(1)$ & Alta \\
\hline $\begin{array}{l}\text { Qualidade de Vida } \\
\text { Escala QoL-AD: score } 13 \text { a } 52 \\
\text { (superior = melhor) } \\
\text { Follow-up: } 18 \text { meses }\end{array}$ & Score médio $=40,02$ & $\begin{array}{l}\text { Diferença média }=0,39 \\
\text { pontos menor neste } \\
\text { grupo (variou de } 1,79 \\
\text { menor a } 1,01 \text { maior) }\end{array}$ & - & $269(1)$ & Moderada $^{2}$ \\
\hline $\begin{array}{l}\text { Qualidade de vida } \\
\text { Escala QoL-AD } \\
\text { Follow-up: } 6 \text { meses }\end{array}$ & Score médio $=39,86$ & $\begin{array}{l}\text { Diferença média }=0,1 \\
\text { pontos menor neste } \\
\text { grupo (variou de } 1,28 \\
\text { menor a } 1,08 \text { maior) }\end{array}$ & - & $332(1)$ & Alta \\
\hline $\begin{array}{l}\text { Saúde mental (depressão) } \\
\text { Escala MADRS: score } 0 \text { a } 30 \\
\text { (inferior = melhor) } \\
\text { Follow-up: } 6 \text { meses }\end{array}$ & Score médio = 1,6 & $\begin{array}{l}\text { Diferença média }=0,1 \\
\text { pontos menor neste } \\
\text { grupo (variou de } 0,74 \\
\text { menor a } 0,54 \text { maior) }\end{array}$ & - & $178(1)$ & Alta \\
\hline $\begin{array}{l}\text { Saúde mental (depressão) } \\
\text { Escala NPI } \\
\text { Follow-up: } 6 \text { meses }\end{array}$ & $\begin{array}{l}\text { Diferença média }=0,1 \\
\text { grupo de intervenção } \\
0,27 \text { maior) }\end{array}$ & $\begin{array}{l}\text { ilos-padrão, superior no } \\
\text { ando de } 0,07 \text { menor a }\end{array}$ & - & $543(2)$ & Alta \\
\hline
\end{tabular}


significativo da suplementação com AG ómega-3 sobre a evolução da gravidade global da demência, quando avaliada pelo Clinical Dementia Rating - Sum of Boxes (CDR-SoB) [DM de 0,00; IC 95\% de -0,58 a 0,57; 542 participantes; dois estudos; evidência científica de alta qualidade] ou sobre a qualidade de vida, quando medida de acordo com a escala Quality of Life Alzheimer's Disease (QoL-AD) [DM de -0,10; IC 95\% de -1,28 a 1,08; 322 participantes; um estudo; evidência científica de alta qualidade]. Aos seis meses, não houve diferença na saúde mental, quando medida de acordo com a escala Montgomery-Åsberg Depression Rating (MADR) [DM de -0,10; IC 95\% de -0,74 a 0,54; 178 participantes; um estudo; evidência científica de alta qualidade] ou com o Neuropsychiatric Inventory (NIP) [DMP de 0,10; IC 95\% de -0,07 a 0,27; 543 participantes; dois estudos; evidência científica de alta qualidade]. Um estudo de pequenas dimensões mostrou benefício da suplementação com AG ómega-3 sobre a independência para as atividades da vida diária, aos 12 meses de tratamento [DM de $-3,50$; IC 95\% de -4,30 a -2,70; 22 participantes; evidência científica de moderada qualidade]. Os estudos incluídos não avaliaram funções cognitivas específicas. A ocorrência de eventos adversos e a sua descrição não foram adequadamente reportadas em nenhum dos estudos incluídos. Dois estudos reportaram que todos os eventos adversos ocorridos foram ligeiros e que não diferiram em frequência entre os grupos de suplementação com AG ómega-3 e os grupos placebo. A análise aos dados de um dos estudos não revelou diferença na frequência da ocorrência de eventos adversos entre o grupo de tratamento e o grupo placebo [Risco relativo (RR) de 1,02; IC 95\% de 0,95 a 1,10; 402 participantes; um estudo; evidência científica de moderada

Tabela 1 - Ácidos gordos poli-insaturados ómega-3 em comparação com placebo na DA ligeira a moderada (continuação)

População: Doentes com DA ligeira a moderada

Intervenção: Suplementos de ácidos gordos poli-insaturados ómega-3

Comparador: Placebo

\begin{tabular}{|c|c|c|c|c|c|}
\hline Medidas avaliadas & \multicolumn{2}{|c|}{$\begin{array}{l}\text { Efeito absoluto antecipado* (95\% IC) } \\
\text { Risco de doença de Alzheimer ligeira a moderada }\end{array}$} & $\begin{array}{l}\text { Efeito } \\
\text { relativo } \\
(95 \% \text { IC) }\end{array}$ & $\begin{array}{l}\text { Número de } \\
\text { participantes } \\
\text { (estudos) }\end{array}$ & $\begin{array}{l}\text { Qualidade } \\
\text { da evidência } \\
\text { (GRADE) }^{\star *}\end{array}$ \\
\hline $\begin{array}{l}\text { Função cognitiva global } \\
\text { Escala ADAS-Cog } \\
\text { (versões variáveis) } \\
\text { Follow-up: } 6 \text { meses }\end{array}$ & \multicolumn{2}{|c|}{$\begin{array}{l}\text { Diferença média }=0,02 \text { desvios-padrão, menor no } \\
\text { grupo de intervenção (variando de } 0,19 \text { menor a } \\
0,15 \text { maior) }{ }^{4}\end{array}$} & - & $566(3)$ & Alta \\
\hline $\begin{array}{l}\text { Função cognitiva global } \\
\text { Escala MMSE: score } 0 \text { a } 30 \\
\text { (superior = melhor) } \\
\text { Follow-up: } 6 \text { meses }\end{array}$ & Score médio $=20,4$ a 22,4 & $\begin{array}{l}\text { Diferença média }=0,18 \\
\text { pontos maior neste } \\
\text { grupo (variou de } 1,05 \\
\text { menor a } 1,41 \text { maior) }\end{array}$ & - & $202(2)$ & Alta \\
\hline $\begin{array}{l}\text { Independência para as } \\
\text { atividades de vida diária } \\
\text { Escala OARS-IADL: score } 0 \text { a } 14 \\
\text { (inferior = melhor) } \\
\text { Follow-up: } 12 \text { meses }\end{array}$ & $\begin{array}{l}\text { Variação do score médio } \\
=4,2\end{array}$ & $\begin{array}{l}\text { Diferença média }=3,5 \\
\text { pontos menor neste } \\
\text { grupo (variou de } 4,3 \text { a } \\
2,7 \text { menor) }\end{array}$ & - & $22(1)$ & Moderada $^{3}$ \\
\hline $\begin{array}{l}\text { Atividades de vida diária } \\
\text { Escalas DAD e ADCS-ADL } \\
\text { Follow-up: } 6 \text { meses }\end{array}$ & \multicolumn{2}{|c|}{$\begin{array}{l}\text { Diferença média }=0,02 \text { desvios-padrão, menor no } \\
\text { grupo de intervenção (variando de } 0,19 \text { menor a } \\
0,16 \text { maior) }\end{array}$} & - & $544(2)$ & Alta \\
\hline $\begin{array}{l}\text { Gravidade da demência } \\
\text { (função cognitiva e combinada) } \\
\text { Escala CDR-SoB: score } 0 \text { a } 18 \\
\text { (inferior = melhor) } \\
\text { Follow-up: } 6 \text { meses }\end{array}$ & Score médio $=6,5$ a 6,75 & $\begin{array}{l}\text { Diferença média }=0 \\
\text { pontos (variou de } 0,58 \\
\text { menos a } 0,57 \text { mais) }\end{array}$ & - & $542(2)$ & Alta \\
\hline Memória & \multicolumn{2}{|l|}{ Não avaliado } & & & \\
\hline
\end{tabular}

IC: intervalo de confiança; Escalas - ADAS-Cog: Alzheimer's disease assessment scale - cognitive subscale; ADCS-ADL: Alzheimer's disease cooperative study - activities of daily living; ADL: activities of daily living; CDR-SoB: clinical dementia rating - sum of boxes; DAD: disability assessment for dementia; IADL: instrumental activities of daily living; NPI: neuropsychiatric inventory; MADRS: Montgomery-Åsberg depression rating scale; MMSE: mini-mental state examination; OARS- IADL: Older Americans Resources and Services Instrumental Activities of Daily Living; QoL-AD: quality of life Alzheimer's disease.

* O risco no grupo de intervenção (e o seu intervalo de confiança a 95\%) é baseado no risco assumido no grupo de comparação e no efeito relativo da intervenção (e o seu intervalo de confiança a $95 \%)$.

** Sistema GRADE:

- Qualidade alta: investigação ulterior é altamente improvável que altere a confiança na estimativa do efeito;

- Qualidade moderada: investigação ulterior tem provável impacto na confiança na estimativa do efeito e pode mesmo alterá-la;

- Qualidade baixa: investigação ulterior tem alta probabilidade de alterar o impacto da confiança na estimativa do efeito e de alterar a própria estimativa do efeito;

- Qualidade muito baixa: grande incerteza na estimativa do resultado.

Desvalorização de um nível por risco importante de viés: medidas avaliadas de forma combinada (i.e. diarreia, quedas, agitação e incluindo variáveis com métodos de avaliação incertos (e.g. vertigens)

Desvalorização de um nível por risco importante de viés: diferente tempo de seguimento entre os grupos.

${ }^{3}$ Imprecisão (intervalo de confiança amplo, apenas 22 participantes, no total).

${ }^{4}$ Diferença média apresentada em alternativa aos valores absolutos nos grupos de intervenção e comparador pois os estudos utilizam diferentes versões da escala.

Diferença média apresentada em alternativa aos valores absolutos nos grupos de intervenção e comparador pois os estudos utilizam diferentes escalas para avaliação da mesma variável. 
qualidade], ou ocorrência de eventos adversos graves [RR de 1,05; IC 95\% de 0,78 a 1,41; 402 participantes; um estudo; evidência científica de alta qualidade] aos 18 meses de tratamento.

Os principais resultados desta RS encontram-se resumidos na Tabela 1.

\section{CONCLUSÃO}

Os autores não encontraram evidência científica convincente para advogar a suplementação com AG ómega-3 no tratamento da DA ligeira a moderada. Este resultado foi consistente para todas as medidas de eficácia estudadas relevantes para os doentes com demência. A ocorrência de eventos adversos com a suplementação com AG ómega-3 pareceu ser baixa, mas com base na evidência científica sintetizada nesta RS, não foi possível extrapolar conclusões sobre a sua tolerabilidade.

Os efeitos da suplementação com AG ómega-3 noutros tipos de demência permanecem por esclarecer.

\section{COMENTÁRIO}

Os AG ómega-3 têm um papel importante na fisiologia humana, nomeadamente através da regulação de processos imunológicos, inflamatórios e hormonais. São também um componente das membranas celulares e têm um papel fundamental no desenvolvimento e normal funcionamento do SNC. ${ }^{1}$ Os AG ómega-3 são considerados essenciais uma vez que a sua síntese endógena não é possível. Algas, óleo de peixe, óleos vegetais e frutos secos são algumas das suas fontes naturais, sendo ainda possível a sua obtenção através de suplementos alimentares. ${ }^{2}$ Tendo em conta a reduzida capacidade de armazenamento destes, é recomendado o seu consumo regular, na dose diária de 250 a 1000 mg, como parte de uma dieta saudável. 3,4

Relativamente ao tratamento da demência, considera-se que, pelas suas propriedades anti-inflamatórias, antioxidantes e importância no normal funcionamento do SNC, o consumo de AG ómega-3 poderia ser benéfico. De forma a esclarecer se o consumo de AG ómega-3 deve ser en- corajado especificamente no tratamento da demência, os autores desta RS pesquisaram e analisaram a evidência científica atualmente disponível, proveniente de estudos experimentais de suplementação com AG ómega-3 em doentes com demência.

Nos três ECAs controlados por placebo incluídos nesta $\mathrm{RS}$, que conferiram uma evidência de qualidade moderada a elevada para a maioria das medidas de eficácia estudadas, não ficou demonstrado benefício significativo do consumo de AG ómega-3 no tratamento da DA ligeira a moderada. As variáveis estudadas ao longo de 6 meses incluíram a função cognitiva global, independência para as atividades da vida diária e gravidade global da doença. Não se registaram eventos adversos significativos em nenhum dos estudos. Não foram encontrados dados relativamente ao efeito da suplementação com AG ómega-3 no tratamento de outras formas de demência.

A ausência de resultados estatisticamente significativos ou clinicamente relevantes e o número reduzido de estudos sobre este tema devem servir de incentivo ao desenho e concretização de EACs que permitam esclarecer definitivamente o papel dos AG ómega-3 no tratamento da DA e outras formas de demência.

\section{IMPLICAÇÕES PARA A PRÁTICA CLÍNICA}

- Relativamente ao tratamento da DA ligeira a moderada, a presente RS encontrou evidência de qualidade moderada a alta relativamente à ausência de efeito benéfico ou ocorrência de eventos adversos da suplementação com AG ómega-3 em doentes com DA.

- Nas outras formas de demência, a evidência científica existente é limitada e são necessários mais estudos para que seja possível extrapolar alguma conclusão.

- A ausência de benefício da suplementação com AG ómega-3 em doentes com DA não deve ter como consequência a adoção, nomeadamente por parte dos profissionais de saúde que lidam com doentes com demência, de comportamentos e atitudes que não promovam uma dieta saudável e equilibrada, incluindo o consumo de AG ómega-3.

\section{REFERENNCIAS}

1. Su HM. Mechanisms of n-3 fatty acid-mediated development and maintenance of learning memory performance. J Nutr Biochem. 2010;21:364-73.

2. World Health Organization. Fats and fatty acids in human nutrition. Report of an expert consultation. [Internet]. FAO Food Nutr. Pap. 2008. [accessed 2017 Sep 12]. Available from: http://www.ncbi.nlm.nih.gov/ pubmed/21812367.

3. EFSA Panel on Dietetic Products Nutrition and Allergies (NDA). Scientific opinion on dietary reference values for fats, including saturated fatty

acids, polyunsaturated fatty acids, monounsaturated fatty acids, trans fatty acids, and cholesterol. Efsa J. 2010;8:1461.

4. Arterburn LM, Hall EB, Oken H. Distribution, interconversion, and dose response of $\mathrm{n}-3$ fatty acids in humans. Am $\mathrm{J}$ Clin Nutr. 2006;83:S1467S-76.

5. Burckhardt M, Herke M, Wustmann T, Watzke S, Langer G, Fink A Omega-3 fatty acids for the treatment of dementia. Cochrane Database Syst Rev. 2016;4:CD009002.

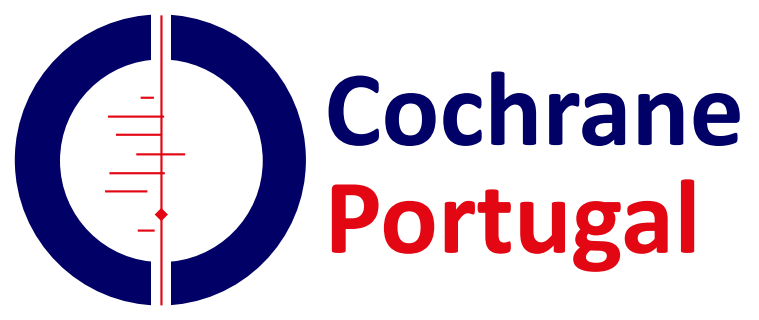

